\title{
Factors Involved In First Myocardial Infarction, Its Complications And Thrombolytic Pattern In Selected Hospitals Of Nepal
}

\author{
${ }^{*}$, K, Shah, A. B. Upadhyaya, L. P. Tibrewala, P. R. Regmi, K. P. Acharya, \\ H. H. Khanal, S. Rajbhandari, D. Shrestha, U. Shrestha, M, Pandey
}

\section{Background}

World Health Organization [WHO] has predicted that by AD 2020 up to three- quarter of death in developing countries would result from non-communicable diseases (NCDs) and that Coronary Heart Disease (CHD) will top the list of killers. Data also indicate that epidemiological transition, which is characterized by aging and changing life style and culminates in epidemics of hypertension (HTN) and CHD, is rapidly occurring in India and other developing countries.

\section{Materials and Methods}

This is a multi centric prospective observational study with five centers in the Kathmandu valley. Peoples are of various regions and multi ethnic in the Kathmandu valley. So this group should form a representative sample of first acute Myocardial infarction (MI) in Nepal. Both sexes and all age group patients of suspected first acute Ml were included in this study. Patients of unstable angina, Old $\mathrm{Ml}$ and second and subsequent $\mathrm{Ml}$ were not included in this study.

\section{Result}

The present study consists of 213 cases of first MI. Male patients were $157(73.7 \%)$ and female patients were $56(26.3 \%)$. Male and female ratio was 2.8: 1.0.Mean Age of first Ml was $57 \pm 11$ years. Younger patients ( $\leq 45$ years) were $42(19.7 \%)$ Hypertensive cases were 94 (44.1\%) and diabetic cases were $62(29.1 \%), 83$ (38.97\%) cases had total serum cholesterol more than $200 \mathrm{mg} \%$, while abnormal serum triglyceride (>150mq\%) found in $111(52.11 \%)$. Positive family history found in $44(20.7 \%)$, Cases of ST-Elevation MI (STEMI) were 192 (90.14\%), Non ST-Elevation MI (NSTEMI) were $19(8.92 \%)$ and New Left Bundle Branch Block (LBBB) were 2 (0.93\%). Only 36 (16.9\%) of patients reached hospitals within 2 hours after onset of chest pain and $117(54.9 \%)$ reached with in 12 
hours. Thrombolysis had done in $95(44.6 \%)$ patients. Only $40(18.78 \%)$ cases were thrombolysed within 30 minutes. Combined in hospital mortality were 16 (7.5\%).

\section{Conclusion}

Mean age of first MI was 57 years, Younger ( $\leq 45 \mathrm{yrs}$ ) population had suffered Ml more $(19.7 \%)$ than what is seen in other parts of the world. Still commonest CHD risk factor was : smoking (72.3\%). HTN (44\% versus $28 \%$ ) and Diabetes Mellitus (29\% versus $14 \%$ ) have increased. Anterior wall $\mathrm{Ml}$ is 1.4 times commoner than inferior wall. Only $17 \%$ of MI patients came within 2 hours to hospital after chest pain. 95 (44.6\%) of STEMI were thrombolysed and only 40 (18.78\%) were thrombolysed within 30 minutes after coming to emergency. In Hospital mortality was $7.5 \%$. Incidence of MI had increased al least 11 times over the past four decades while population of Kathmandu valley has increased by three times. 